\title{
Ultrasound measurement learning of fetal sex during the first trimester: does the experience matter?
}

\author{
Ricardo Savirón- \\ Cornudella' \\ Isabel González-Ballano' \\ Ana Cisneros-Gimeno² \\ Diego Lerma-Puertas' \\ Pilar Pérez-Pérez' \\ Paola Montañés-Bello' \\ Juan de León-Luis ${ }^{3}$ \\ 'Department of Prenatal Diagnosis \\ and Obstetrics Ultrasound, \\ Obstetrics and Gynaecology, \\ Hospital Universitario Miguel Servet, \\ ${ }^{2}$ Anatomy and Histology Department, \\ University of Zaragoza, Zaragoza, \\ Spain; ${ }^{3}$ Department of Prenatal \\ Diagnosis and Obstetrics Ultrasound, \\ Obstetrics and Gynaecology, \\ Hospital General Gregorio Marañón, \\ Universidad Complutense de Madrid, \\ Madrid, Spain
}

This article was published in the following Dove Press journal:

Research and Reports in Focused Ultrasound

15 December 2015

Number of times this article has been viewed

Objective: To determine the probability of correctly diagnosing fetal sex during the first trimester by ultrasound according to crown-rump length (CRL) and previous ultrasound experience.

Methods: A cohort study was performed from March 2012 to April 2013. The 2,314 first trimester pregnancy ultrasounds were examined. Eight sonographers, according to previous ultrasound experience, were divided into two groups: senior and junior. For fetal sex estimation, the method of a sagittal section and the relation between the angle formed by the genital tubercle and spinal column was used.

Results: In 1,986 cases, fetal sex was diagnosed, with a success rate of $90.1 \%$. A directly proportional relationship between the rate of success in fetal sex diagnosis and CRL $(P<0.001)$ was described. The rate of success in male fetuses was significantly higher than in female fetuses $(94.6 \%$ vs $86.3 \%$, respectively), $P<0.001$. In the senior sonographers group, the rate of overall success was $89.2 \%$ vs $90.5 \%$ in the junior group, the difference not being significant. The experience of sonographers did not reach statistical significance ( $95 \%$ confidence interval: $0.871-1.031 ; P=0.213)$.

Conclusion: The prediction of fetal sex over $65 \mathrm{~mm}$ of CRL in both sexes is approximately $95 \%$ and from $76 \mathrm{~mm}$ onward is $99 \%$. Both CRL and fetal sex influence the rate of success; however, the experience of a sonographer has not proven to be an influential factor. These results could be explained because the technique for diagnosing sex during the first trimester is very simple to learn.

Keywords: fetal sex, first trimester, ultrasonography, prenatal diagnosis

\section{Introduction}

Improvements in ultrasound systems have allowed over the last few years to see the fetal morphology more clearly, and to detect fetal sex earlier during the first trimester, especially for increasing embryo size, and for male fetuses. ${ }^{1,2}$ The early diagnosis of fetal sex during the first trimester of pregnancy, non-invasively and affordably may be important in the diagnosis of sex-linked diseases.

At the beginning of the 5th week of pregnancy, a pair of swelling or cloacal folds form on both sides of the cloacal membrane, which joins in front of the membrane to form a thickening in the midline called the genital tubercle (primordium penis or clitoris). During the 9th week, they begin to differentiate, but the similar appearance of the external genitalia in embryos of both sexes may persist until the 9th week of development. 3,4

Until now, published literature brings inconsistent data regarding the rate of success in the diagnosis of fetal sex during the first trimester, although most of the articles

Correspondence: Ricardo SavirónCornudella

Unidad de Ecografías Obstétricas y Diagnóstico Prenatal, Servicio de Obstetricia y Ginecología, Hospital Universitario Miguel Servet, C/lsabel La Católica 3, 50009 Zaragoza, Spain Tel +34976765 500

Email rsaviron@gmail.com 
agree that it depends on crown-rump length (CRL) and fetal sex. Various methodologies have been reported to describe the fetal sex during the first trimester. ${ }^{5}$ In 1990, Bronshtein et al described the "dome sign", an image of the scrotum and penis directed cranially in male fetuses and an image of two or four parallel lines representing the clitoris directed caudally. ${ }^{6}$ In 2000, Pedreira proposed three echogenic points (triangle-shaped) in male fetuses. In female fetuses, however, only two echogenic lines corresponding to the clitoris are identified. This technique has an accuracy of $82.1 \% .^{7}$ Finally, in 2006, Efrat et al assign male sex when the angle of the genital tubercle to a horizontal line through the surface of lumbosacral skin is $>30^{\circ}$ and female sex when the genital tubercle is parallel or convergent $\left(<30^{\circ}\right)$ to the horizontal line. This method achieved an overall accuracy of $98.6 \%{ }^{8}$

The aim of this study was to determine the probability of correctly diagnosing fetal sex during the first trimester according to CRL and the sonographers previous ultrasound experience.

\section{Patients and methods}

A cohort study was performed with prospective follow-up of pregnant women attending the Prenatal Diagnosis Unit to perform an ultrasound study for obtaining combined first trimester screening during the period from March 2012 to April 2013. The study protocols were approved by the Ethical Committee for Clinical Research of Aragón (CEICA), Aragón, Spain. Oral informed consent was obtained from all patients. Each pregnant woman was evaluated by one of eight sonographers who applied the correct algorithm to determine fetal sex. Inclusion criteria for patients were singleton pregnancy, a viable fetus without congenital anomalies, and CRL between 45 and $80 \mathrm{~mm}$. The exclusion criteria included patient refusal to continue the study, attended birth at another hospital and the diagnosis of fetal death, congenital anomaly, or inability to confirm fetal sex at birth. The examinations were performed with Voluson GE 730 Expert, Voluson GE E6, and Voluson GE E8.

An initial assessment of the type of gestation, vitality, embryo morphology, and possibility to describe the fetal sex without telling the patient was performed. The assessment of fetal sex and CRL by ultrasound was performed according to the following guidelines: perform a sagittal section with a two-dimensional sonograph (a three-dimensional sonograph is not necessary) with the fetus in neutral position, visualizing the whole fetus (with the greatest expansion possible) to assess the fetal position, neither too flexed nor too extended and measuring the angle formed by the genital tubercle and the spinal column and collect the following, ${ }^{8}$ male sex: genital tubercle with cranial notch

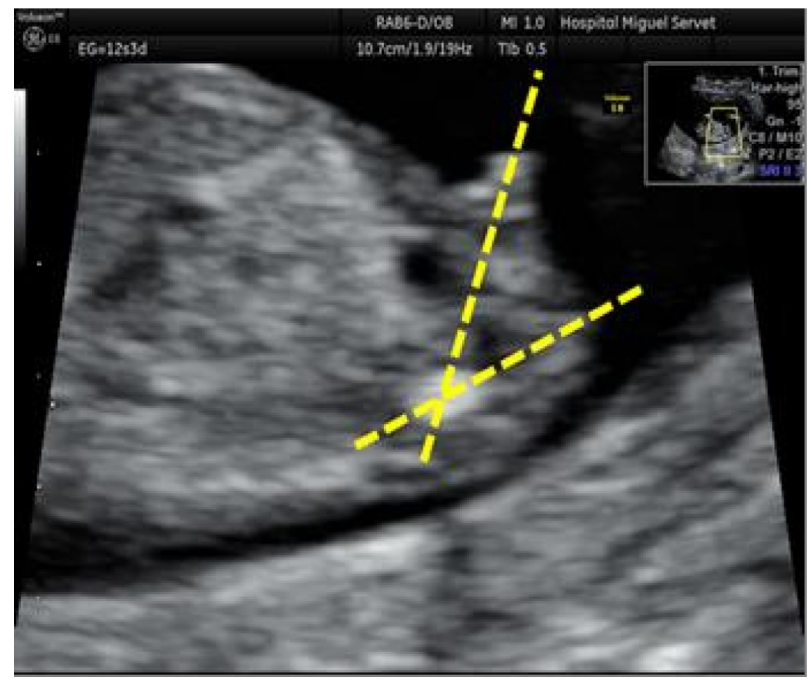

Figure I Male sex: ultrasound identification of the male sex at the first trimester ultrasound scan shows tubercle's angle $>30^{\circ}$.

(angle $>30^{\circ}$ relative to the spinal column; Figure 1), female sex: genital tubercle with caudal notch (angle $<10^{\circ}$ relative to the spinal column; Figure 2), and undetermined sex because of fetal position or angle between $10^{\circ}$ and $30^{\circ}$. It is very important that the fetus is visualized in the neutral position, otherwise the angle may be overestimated or underestimated and the result may be misleading. Transabdominal and/or transvaginal ultrasound investigations were performed to obtain a correct sagittal section. After giving the guidelines for the detection of fetal sex (in a prior training) and according to previous ultrasound experience, two groups of sonographers were separated as senior $(>10$ years, experience) and junior ( $<5$ years, experience).

The predictor or independent variables include embryo's CRL, fetal position (suitable, not assessable for unfavorable fetal position or not assessable for angle between $10^{\circ}$ and

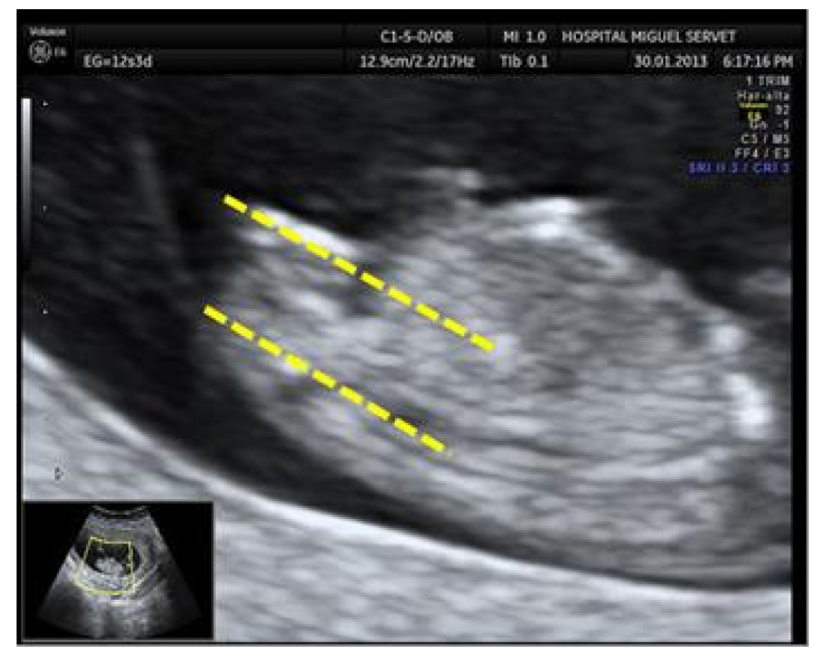

Figure 2 Female sex: ultrasound identification of the female fetal sex at first trimester ultrasound scan shows the genital tubercle parallel to the spinal column. Note: Inset shows full ultrasound scan. 
$30^{\circ}$ ) and sonographer (qualified by experience). The outcome variable was described by ultrasound fetal sex. A second outcome variable is secondary to obtaining rightness or wrongness (true or false) as matching or not sex described by first trimester ultrasound and sex at birth.

The collection of variables was performed in two stages, recruitment coinciding with the ultrasound examination during the first trimester and post-delivery. Perinatal data were obtained for eight obstetricians blinded to the results of the variables display during the first trimester ultrasound. Records of newborns were created to collect perinatal data. All data were collected in coded form and were entered into a database designed for this purpose. The statistical package used was SPSS 20.0 (IBM Corporation, Armonk, NY, USA). A value of $P<0.05$ was considered significant, considering the confidence intervals (CIs).

To describe the characteristics of the pregnant women included in the study, predictor and outcome variables were evaluated descriptively. Qualitative variables are detailed as a percentage. Quantitative variables are detailed with centralization parameters (mean/median) and scattering parameters (standard deviation, interquartile range, or other). To check the fit to normal of each of the variables analyzed in this study, the Kolmogorov-Smirnov test was used.

The possible association (depending on the degree of dependence/independence) between some predictors and outcome variables was studied with the aim of responding to the analysis that takes place during the inference phase.
Chi-square and logistic regression according to variable types using bivariate and multivariate analysis were used.

\section{Results}

Between March 2012 and April 2013, 2,314 first trimester pregnancy ultrasounds were examined. A total of 345 patients who aborted after the first trimester ultrasound or the patients who gave birth at another hospital were not included in the sample (14.9\%). The average age of pregnant women was 32.36 years (15-44 years) and the mean gestational CRL of the first trimester ultrasound was $61.86 \mathrm{~mm}$, with a median of $62 \mathrm{~mm}$ and a standard deviation of $7.57 \mathrm{~mm}$. Mean days to delivery (adjusted CRL) was 278 (162-299 days).

In 328 cases $(14.2 \%)$, it was not possible to diagnose due to unfavorable fetal position or intermediate angle of the genital tubercle (between $10^{\circ}$ and $30^{\circ}$ ). The outcome was confirmed at delivery without encountering any case of ambiguous genitalia. In 1,986 cases $(85.8 \%)$, the fetal sex was diagnosed with a success rate of $90.1 \%$ (1,789 cases) and failure rate of $9.9 \%$ (197 cases). A directly proportional relationship between the rate of success in fetal sex diagnosis and CRL $(P<0.001)$ was described. With a CRL greater than $65 \mathrm{~mm}$, the rate of success is over $95 \%$ and for CRL $77 \mathrm{~mm}$ or longer it is close to $100 \%$.

Analyzing the rate of success in the diagnosis of fetal sex, it was found that fetuses were significantly higher in males than in females $(94.6 \%$ vs $86.3 \%$, respectively), $P<0.001$ (Figure 3).

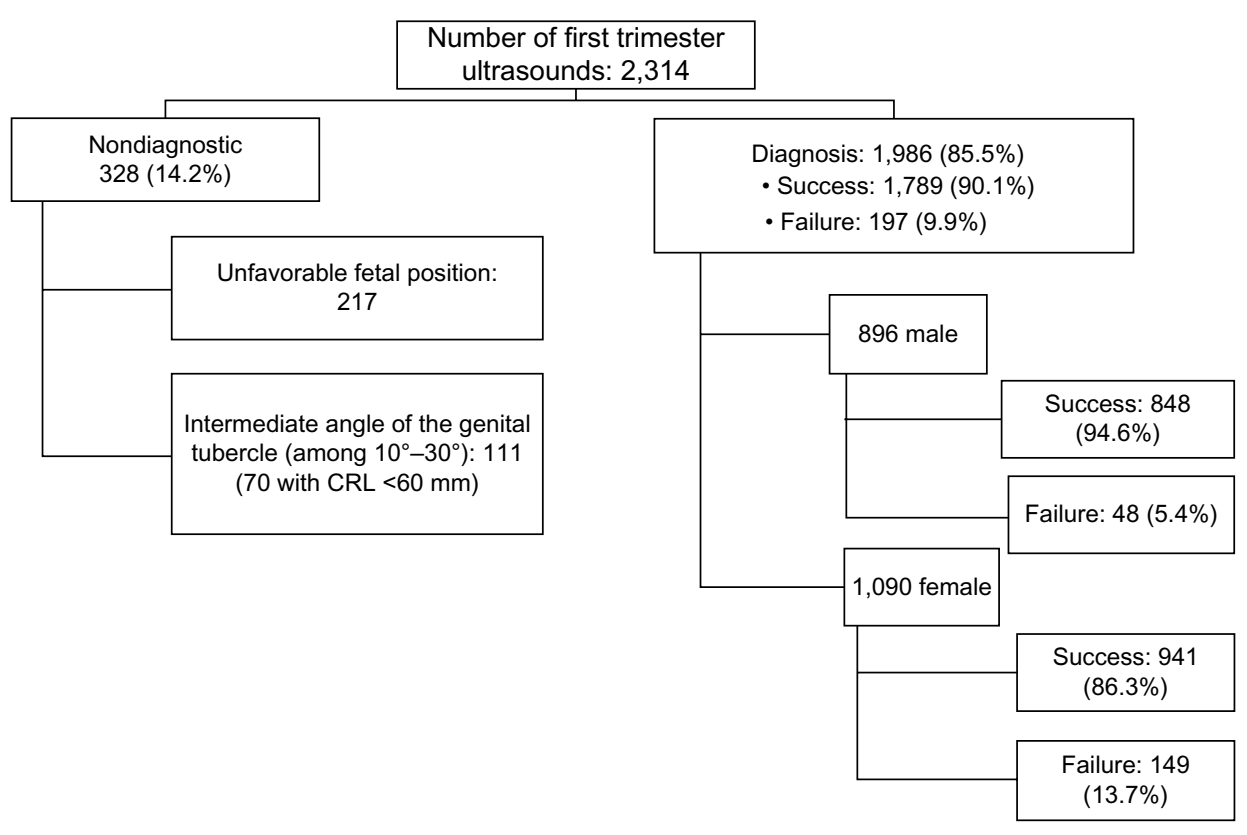

Figure 3 Success rate according to fetal sex algorithm. Abbreviation: CRL, crown-rump length. 
Analyzing the association between the rate of success in the diagnosis of fetal sex and the CRL, it was noted that in male fetuses compared with female fetuses, the rate of success is higher for short CRL. For example, in male fetuses with $51 \mathrm{~mm}$ of CRL, the rate of success is $93 \%$, and this rate is achieved only in female fetuses with a CRL of $66 \mathrm{~mm}$. The rate of success below $51 \mathrm{~mm}$ in both male and female fetuses is below $80 \%$ ( $68 \%$ and $76 \%$, respectively). However, from $66 \mathrm{~mm}$ the rate of success for both sexes is very similar and greater than $95 \%$ (Figure 4 ).

The average rate of success in the fetal sex diagnosis for all cases was $90.1 \%$. The total number of cases was 2.37 times higher in the junior sonographers group. However, in the senior sonographers group, the rate of overall success was $89.2 \%$ vs $90.5 \%$ in the junior group, the difference not being significant.

Finally, Table 1 describes the results of univariate and multivariate analysis of independent variables and its relation to the success rate in the diagnosis of fetal sex. Consistently, both the CRL with an odds ratio of 1.131 (95\% CI: 1.100$1.640 ; P<0.001)$, and fetal sex with an odds ratio of 2.197 (95\% CI: 1.469-3.286; $P<0.001$ ) were significant. The experience of sonographers did not reach statistical significance (95\% CI: $0.871-1.031 ; P=0.213$ ).

\section{Discussion}

Factors that have been analyzed are the experience of the sonographer and fetal CRL determining the accuracy of the estimation of fetal sex using the method of a sagittal section and the relation between the angle formed by the genital tubercle and spinal column.

Previously, other authors with different methodologies had studied the rate of success in the diagnosis of fetal sex based on gestational age and/or fetal sex. Whitlow et al combined fetal sex identification in a sagittal and transverse plane with an accuracy that increases with increasing gestational age. The global success rate was $85 \%{ }^{9}$ Similarly, Hsiao et al combined both planes for the detection with a success rate of fetal sex diagnosis of $91.8 \%$ and also increasing with age gestational. ${ }^{10}$ Chelli et al using the same methodology described in our study achieved a success rate of $85.7 \% .^{11}$

In our study, the rate of success in fetal sex diagnosis following the methodology described by Chelli et al was $90.1 \%$. This rate was significantly associated with the CRL, and it increases in male fetuses. The rate of success was higher in male fetuses than in female ones, $94.6 \%$ compared with $86.3 \%$. Adiego Burgos et al also had similar results with a success rate in male fetuses of $90.6 \%$ and $83.1 \%$ in females. ${ }^{12}$ Only Mazza et al had different results with a success rate of $100 \%$ in female determination and $46 \%$ in male fetuses. ${ }^{13}$ Relative to the CRL and fetal sex, these data are confirmed, being the earlier diagnosis in male fetuses. ${ }^{13}$

Regarding the size of the fetus, according to the CRL, it is clear that there is a direct correlation between diagnostic accuracy and CRL regardless of fetal sex. ${ }^{14}$ Our data demonstrate that in male fetuses diagnosed with CRL below $65 \mathrm{~mm}$, the rate of success is statistically higher than in female fetuses. In all studies, including ours, the ultrasound assignation increases with gestational age and the rate of success is near $100 \%$ from $11+6$ weeks of gestation. ${ }^{1}$ These results are in agreement with classic embryology, which establishes that differential development (female/male) starts after 50 $\mathrm{mm} \mathrm{CRL}$ is reached (9 weeks). ${ }^{13}$

No significant differences in the rate of success of fetal sex diagnosis depending on the experience of the sonographer were found. Finally, in the multivariate analysis, these data

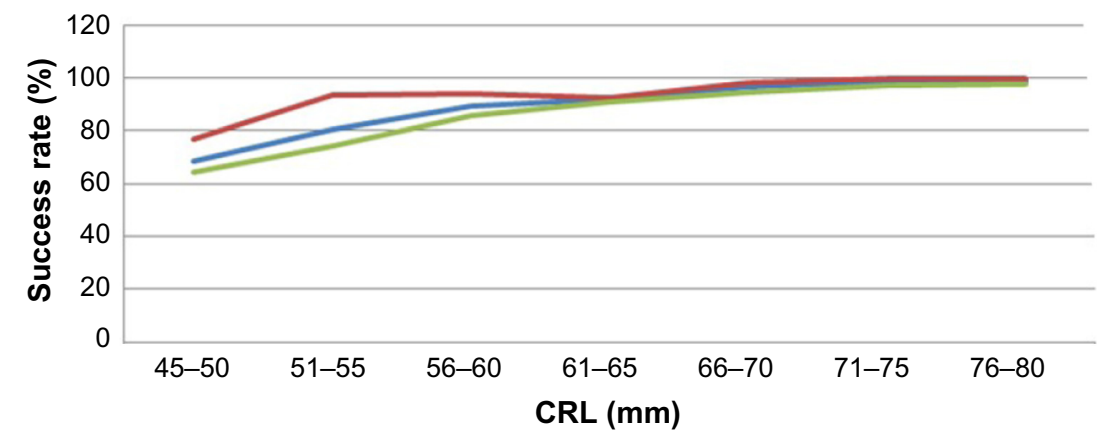

\begin{tabular}{|l|l|l|l|l|l|l|l|}
\hline Global & 68.7 & 80.6 & 89.2 & 91.8 & 96.6 & 98.8 & 99 \\
\hline Male & 76.9 & 93.5 & 94.1 & 92.5 & 98.4 & 100 & 100 \\
\hline Female & 64.5 & 74.3 & 85.8 & 91.2 & 94.7 & 97.4 & 98 \\
\hline
\end{tabular}

Figure 4 Global success rate according to CRL and divided by sex. Abbreviation: CRL, crown-rump length. 
Table I Results of univariate and multivariate analysis of independent variables and its relation to the success rate in the diagnosis of fetal sex

\begin{tabular}{|c|c|c|c|c|}
\hline & \multirow[t]{2}{*}{ OR } & \multicolumn{2}{|l|}{$95 \% \mathrm{Cl}$} & \multirow[t]{2}{*}{$P$-value } \\
\hline & & Lower & Higher & \\
\hline \multicolumn{5}{|l|}{ Univariate } \\
\hline CRL (mm) & 1.140 & 1.109 & 1.172 & $<0.001$ \\
\hline Sonographer's experience & 1.064 & 0.766 & 1.479 & $0.7 I I$ \\
\hline Male fetus & 2.744 & 1.862 & 4.044 & $<0.001$ \\
\hline \multicolumn{5}{|l|}{ Multivariate } \\
\hline CRL (mm) & 1.131 & 1.100 & 1.640 & $<0.001$ \\
\hline Sonographer's experience & 0.948 & 0.871 & 1.031 & 0.213 \\
\hline Male fetus & 2.197 & 1.469 & 3.286 & $<0.001$ \\
\hline
\end{tabular}

Abbreviations: $\mathrm{Cl}$, confidence interval; $\mathrm{CRL}$, crown-rump length; $\mathrm{OR}$, odds ratio.

are consistent and the experience of the sonographer does not influence the probability of success.

There have been no previous studies on whether learning technique or the experience of the sonographer influences the rate of success of fetal sex determination. Our results show with the largest sample studied to date that 1-week training is enough to make a correct diagnosis, thanks to the good visualization of equipment, the simplicity of viewing genital tubercles and usually the fetus being placed on the back.

In summary, although in $15 \%$ of cases fetal sex cannot be estimated because of fetal position or intermediate genital tubercle's angle, the prediction of fetal sex over $65 \mathrm{~mm}$ of CRL in both sexes is approximate $95 \%$ and from $76 \mathrm{~mm}$ is $99 \%$. Of the three variables that have been analyzed, it was found that CRL and fetal sex do influence the rate of success; however, the experience of the sonographer has not been shown to be an influential factor. These results could be explained because the technique for diagnosing sex during the first trimester is very simple to learn. Therefore, knowing the methodology, with a short learning curve and ultrasound equipment available today, we have a simple and practical tool to establish fetal sex during the first trimester with great reliability.

\section{Acknowledgment}

The authors are grateful for editorial assistance.

\section{Disclosure}

The authors report no conflicts of interest in this work.

\section{References}

1. Hyett JA, Gardener G, Stojikovic-Mikie T, et al. Reduction in diagnostic and therapeutic interventions by non-invasive determination of fetal sex in early pregnancy. Prenat Diagn. 2005;25:1111-1116.

2. Lubusky M, Studnickova M, Skrivanek A, Vomackova K, Prochazka M. Ultrasound evaluation of fetal gender at 12-14 weeks. Biomed Pap Med Fac Univ Palacky Olomouc Czech Repub. 2012;156(4):324-329.

3. Larsen W. Embriologia Humana. 3rd ed. Madrid: Elsevier; 2003.

4. Moore K. Embriologia Clínica. Madrid: Elsevier Saunders; 2013

5. Emerson DS, Felker RE, Brown DL. The sagittal sign. An early second trimester sonographic indicator of fetal gender. J Ultrasound Med. 1989; 8:293-297.

6. Bronshtein M, Rotten S, Yoffe N, Blumenfeld Z, Brandes JM. Early determination of fetal sex using transvaginal ultrasonography: technique and pitfalls. J Clin Ultrasound. 1990;18:302-306.

7. Pedreira DAL. In search for the "third point". Ultrasound Obstet Gynecol. 2000;15:262-263.

8. Efrat Z, Perri T, Ramati E, Tugendreich D, Meizner I. Fetal gender assignment by first trimester ultrasound. Ultrasound Obstet Gynecol. 2006;27:619-621.

9. Whitlow BJ, Lazanakis MS, Economides DL. The sonographic identification of fetal gender from 11 to 14 weeks of gestation. Ultrasound Obstet Gynecol. 1999;13(5):301-304.

10. Hsiao CH, Wang HC, Hsieh CF, Hsu JJ. Fetal gender screening by ultrasound at 11 to $13+6$ weeks. Acta Obstet Gynecol Scand. 2008;87: 8-13.

11. Chelli D, Methni A, Dimassi K, et al. Fetal sex assignment by first trimester ultrasound: a Tunisian experience. Prenat Diagn. 2009;29(12): $1145-1148$.

12. Adiego Burgos B, Martínez Ten P, Pérez Pedregosa J, et al. Determinación del sexo fetal en el primer trimestre de la gestación: estudio prospectivo. Rev Chil Obstet Ginecol. 2010;75(2):117-123. Spanish.

13. Mazza V, Falcinelli C, Paganelli S, et al. Sonographic early fetal gender assignment: a longitudinal study in pregnancies after in vitro fertilization. Ultrasound Obstet Gynecol. 2001;17:513-516.

14. Benoit B. Early fetal gender determination. Ultrasound Obstet Gynecol. 1999;13:299-300.
Research and Reports in Focused Ultrasound

\section{Publish your work in this journal}

Research and Reports in Focused Ultrasound is an international, peerreviewed, open access online journal publishing original research, study protocols, reports, editorials, reviews and commentaries on al aspects of focused ultrasound technologies for the treatment of cancer and other diseases. The manuscript management system is completely

\section{Dovepress}

online and includes a very quick and fair peer-review system, which is all easy to use. Visit http://www.dovepress.com/testimonials.php to read real quotes from published authors. 